and finger function; dynamometry; gross and pinch grip measured using the Jamar dynamometer; the Jebson-Taylor Hand Function Test and goniometry including joint ranges for wrist, thumb and individual fingers of each hand. Ethical approval was granted from the Local Research Ethics Committee.

Results 67 patients with a confirmed diagnosis of RA entered into the study. There were no statistically significant differences in baseline characteristics or demographic data. 52 patients completed all visits for all measurements. 58 attended at their one month measurement and 54 at their 3 month measurement. 15 subjects dropped out of the study. There was no significant difference in drop out rates between the 3 groups. One subject was lost to follow up. Analysis was performed using the SPSS package version 10.0. Differences in change between baseline and follow-up at 6 months were assessed using ANOVA for approximately normal data and Kruskal-Wallis test for non-normal data. At 6 months there was a statistically significant difference in AIMS II Upper limb function ( $p=0.007$, F-ratio 5.47) in the 3 groups. Comparison of pairs of groups at the 5\% level of significance with Bonferroni adjustment showed that Group 1 was significantly different from Groups 2 and 3. In addition there were non-significant differences but improvements noted in AIMS (hand and finger function subscales) and pinch grip for both hands in this group. There were no changes in hand function or impairment measures in Groups 2 or 3.

Conclusions This study demonstrates a statistically and clinically significant improvement in upper limb function following a programme of home strengthening hand exercises in patients with RA. Further work is recommended to evaluate which specific exercises are most beneficial.

\section{HP0007 THE HAQ INDEX - RELATIONS TO DIFFICULTY IN DAILY ACTIVITIES}

${ }^{1} \mathrm{KU}$ Nordh, ${ }^{2} \mathrm{U}$ Nordenskiöld. 'Department of Occupational Therapy, Division of Rheumatology, Karolinska Hospital, Stockholm, Sweden; ${ }^{2}$ Department of Occupational Therapy and Rehabilitation Medicine, Sahlgrenska Universitetssjukhuset, Gothenburg, sweden

\subsection{6/annrheumdis-2001.1247}

The objectives were to make an analysis of the HAQ disability index concerning if the patients marked their difficulty in the answere alternatives "no, any or much difficulty" and "using assistive devices" as well as if their assistive devices were in use at home.

The methods One hundred and eighteen patients, with rheumatoid arthritis have attended in the program "Early Arthritis" which all have been at their two year control at Karolinska hospital. One hundred and eighteen of 124 patients were included in this study, 30 male and 88 female with the mean age of 53 years. The patients have marked their perceived difficulty in the HAQ seven times during two years and filled in a checklist with assistive devices. In the HAQ the scale steps are $0=$ no difficulty, 1 = with any difficulty, 2 = with much difficulty, $2=$ with assistive devices and $3=$ unable to do. Both "much difficulty" and "using assistive devices" are scored with the scale step 2.

The results showed that 99 of 118 patients had assistive devices at home, 20 men had 4.6 assistive devices and 79 women had 10 assistive devices per person. In spite of that $97 \%$ of the devices were in use by the men and $95 \%$ were in use by the women, seventeen men and 57 women had never filled in the answere alternative "with assistive devices" in the HAQ, even though they were using the devices. If the men and the women had filled in the alternative "with assistive devices", an increased HAQ index should have been identified. The mean value had increased from 0,28 to 0,5 for the men and for the women the mean value had increased from 0,56 to 0,89 .

The conclusion The HAQ index is not reliable enough to show the level of patients perceived difficulty in daily activities in relation to using assistive devices. The scale step 2 is used for both "much difficulty" and for "with assistive devices" and the patients in this study had not filled in the alternative "with assistive devices". The HAQ index mixes two different aspects of disability, dependence in terms of use of assistive devices and perceived difficulty.

\section{REFERENCES}

1 Fries JF. The assessment of disability; from first to future principles. $\mathrm{Br} J$ Rheumatol. 1983; 22(3 Suppl):48-58

2 Guillemin F, Briacon S, Pourel J. Validity and discriminant ability of HAQ Functional Index in early rheumatoid arthritis. Disabil Rehabil.1992;14:71-7

3 Tennant A, Hillman M, Fear J. Are we making the most of The Stanford Health Assessment Questionnaire? Br J Rheumatol. 1996;35:574-8

4 Nordenskiöld U, Grimby G. Assessment of disability in women with rheumatoid arthritis in relation to grip force and pain. Disabil Rehabil. 1997;19:13-9

\section{HP0008 THE USE OF COMPLEMENTARY AND ALTERNATIVE MEDICINE IN PATIENTS WITH RHEUMATOID ARTHRITIS}

${ }^{1} \mathrm{AC}$ Seymour, ${ }^{1} \mathrm{CA}$ Mayes, ${ }^{1} \mathrm{KA}$ Young, ${ }^{2} \mathrm{~S}$ Stafford, ${ }^{3} \mathrm{~L}$ Waterhouse, ${ }^{4} \mathrm{H}$ Dart. ${ }^{1}$ Rheumatology, St Albans City Hospital, St Albans; ${ }^{2}$ Rheumatology, Winchester and Eastleigh, Winchester; ${ }^{3}$ Rheumatology, Selly Oaks, Birmingham; ${ }^{4}$ Rheumatology, Robert Jones and Agnus Hunt, Oswestry, UK

\subsection{6/annrheumdis-2001.1248}

Background There are few randomised-controlled studies on the efficacy of Complementary/Alternative Medicine (CAMs). Lack of or misleading information for patients could lead to inappropriate management. There is little liaison or accountability between medical and CAM practitioners in the UK and little information on referral patterns and extent of knowledge about CAMs amongst rheumatologists. For this reason there may be a need for a structured departmental policy.

Aims To assess the number, type and cost of CAMs used by patients with Rheumatoid Arthritis (RA).

Methods A postal questionnaire was sent to 200 patients who are still attending The Early Rheumatoid Arthritis Study (ERAS), a long term observational study of early RA from 9 different regions of England. The ERAS database holds details of all drug therapies and outcomes on patients for up to 13 years follow up. The responses of the first $100(\mathrm{n}=33 \mathrm{M}, 67 \mathrm{~F})$ patients are reported here.

Results 76 patients had used or were currently using CAMs (currently using $\mathrm{n}=35, \mathrm{n}=41$ have used). Reasons for their use were:- Symptom relief $(\mathrm{n}=30)$, GP advice $(\mathrm{n}=2)$, Rheumatologist advice $(\mathrm{n}=2)$, wanting to find alternatives to Rheumatology drugs $(n=26)$, other $(n=9)$ and not recorded $(n=4)$. The most widely used CAMs were:- vitamin supplements $(\mathrm{n}=$ $53,73 \%)$, Homeopathy $(\mathrm{n}=30,41 \%)$, copper bracelet $(\mathrm{n}=$ $31,42 \%)$, Acupuncture $(\mathrm{n}=33,45 \%)$, Osteopathy $(\mathrm{n}=$ $29,40 \%)$, Aromatherapy ( $\mathrm{n}=19,26 \%)$, cod liver oil $(\mathrm{n}=19$, $26 \%)$ and multivitamins $(n=17,23 \%) .54$ patients used more than one. Cost to each patient over the last year:- $<£ 15$ (n = 14), $£ 15-£ 50(\mathrm{n}=16), £ 50-£ 100(\mathrm{n}=6),>100(\mathrm{n}=8)$ and not recorded $(n=29)$. Of the 35 patients currently on CAMs, 22 
were still on, and 6 had used second line (disease modifying) drugs.

Conclusion Our results show that $76 \%$ of RA patients were or had taken CAMs. These were in addition to second line (disease modifying) drugs, and not as alternatives. A few CAMs (e.g. acupuncture) are provided by some Health Service Hospitals, but generally were paid for by patients. The main reason for stopping were lack of efficacy and expense.

\section{SP0171 PATIENT PRIORITIES AT THEIR FIRST CONSULTATION}

SC Stafford, NL Cox. Rheumatology, Royal Hampshire County Hospital, Winchester, UK

\subsection{6/annrheumdis-2001.1249}

Background In the changing world of today's healthcare, it has now been recognised that the provision of services should allow patients participation and their say in priority setting. Patients are now much better informed about their disease, treatment available, and the quality of care to which they are entitled. To improve satisfaction we have to identify the goals and expectations for each patient as these may well differ from those the clinician or health professional may think them to be.

Objectives To further explore these concepts and to identify the goals and expectations of each individual patient when they attended for their first consultation in the Rheumatology OutPatient Department.

Method A questionnaire was designed and sent to patients for completion prior to their attendance at their first consultation. Four domains were selected and the patients were asked to prioritise them in order of their goals and expectations from this consultation.

1) Pain Relief 2) Diagnosis 3) Outlook 4) Help/Other. An extra option was included for individual comments. 500 fully completed questionnaires were randomly selected from the patient notes in the clinic. The order of priority, patient's age, gender and diagnosis were recorded. The results were analysed by variance.

Results The study consisted of 166 male and 334 female patients. Their overall First Choice of the categories was - Pain Relief 235(47\%) Diagnosis 197(39.5\%) Outlook 43(8.5\%) Help/ other 25(5\%).

These results varied by Gender, Age, Inflammatory and Degenerative, and to a lesser degree by Soft Tissue and Mixed Connective Disease.

Conclusion The results suggest that overall relief of pain is of prime importance, especially amongst the elderly and those with R. A. Diagnosis is the main priority for those $<40$ years and male patients with O. A. Outlook and Practical Help maybe of concern later but is rarely a priority at first consultation.

\begin{tabular}{llll}
\multicolumn{4}{l}{ Abstract SP0171 Table 1 } \\
\hline First Choice & Overall & Age & Rheumatoid Arthritis \\
& M F & $<40$ yrs > $\mathbf{6 0}$ yrs & M F \\
\hline Diagnosis & $38 \% 40 \%$ & $52 \% 41 \%$ & $39 \% 35 \%$ \\
Outlook & $11 \% 8 \%$ & $5 \% 8 \%$ & $12 \% 10 \%$ \\
Pain Relief & $49 \% 46 \%$ & $38 \% 46 \%$ & $45 \% 52 \%$ \\
\hline
\end{tabular}

Results of Patient's First Choice Selections.

\section{Psychological aspects and management}

\section{HP0023 MANAGING THE IMPACT OF OSTEOARTHRITIS AMONG RETIRED PROFESSIONAL FOOTBALLERS: AN INTERPRETATIVE PHENOMENOLOGICAL ANALYSIS}

AP Turner, JH Barlow. Psychosocial Research Centre, Coventry University, Coventry, UK 10.1136/annrheumdis-2001.1250

Background It has been argued that retirement from professional sport poses unique psychosocial challenges. These challenges may be exacerbated if retirement is accompanied by long-term health problems.

Objective The purpose of this study was to explore the experience of living with osteoarthritis (OA) from the perspective of retired professional footballers in the UK.

Methods Data were gathered via cross-sectionaI unstructured interviews. It is recognised that the "subtleties" and "personal meanings" inherent in chronic illness are best investigated using qualitative methods. Five retired-professional footballers between the ages of 61 and 68 years were interviewed about the changes experienced as a result of their OA and how they managed these changes. Interviews were transcribed verbatim and analysed using Interpretative Phenomenological Analysis (IPA).

Results Living with OA involved pain, surgery, medication and restricted mobility. Feelings of frustration were often associated with disruption to work, social and leisure activities. Three broad themes emerged which encapsulated respondents' strategies to manage their OA-related problems: comparing self with former self and others; adapting lifestyle and positive reappraisal.

Conclusion All of the retired footballers used downward social comparison to manage the emotional turmoil associated with OA. For some this entailed comparing themselves with other retired players and served to reinforce feelings that they had nothing serious to complain about. Respondents continued to perform modified exercise and leisure activities, but were mindful of aggravating painful symptoms. Respondents demonstrated a positive attitude towards their condition. This was evident through the use of humour as a strategy for managing their OA.

\section{SP0139 'A NEW LEASE OF LIFE': THE EXPERIENCES OF OLDER VOLUNTEERS TRAINED TO DELIVER AN ARTHRITIS SELF- MANAGEMENT COURSE}

JM Hainsworth, JH Barlow, AP Turner. Psychosocial Research Centre, Coventry University, Coventry, UK

\subsection{6/annrheumdis-2001.1251}

Background The value of volunteerism among older people continues to be recognised, with benefits extending to both giver and recipient. The purpose of this study was to determine whether conducting an arthritis self-management course as a volunteer lay-leader would lead to improvements in terms of physical and psychological health status.

Methods The study was a pre-test post-test design with data collected at three points in time; before attending training, 6 weeks, and 6 months after training. Data were collected through selfadministered postal questionnaires and telephone interviews. The sample comprised 22 older people with arthritis. The majority of participants were women $(\mathrm{N}=20)$, with either osteoarthritis $(\mathrm{N}=14)$ or rheumatoid arthritis $(\mathrm{N}=9)$, a mean age of 